International Egyptian Journal of Nursing Sciences and Research

(IEJNSR)

Review article

Received June 8, 2020

Revised July 9, 2020

Published July 16, 2020

\title{
Diabetes Mellitus and Women's Health
}

\author{
Tyseer Marzouk \\ Woman's Health and Midwifery Department, Faculty of Nursing-Mansoura University, Egypt \\ Corresponding author: tm_fathy@yahoo.com
}

\begin{abstract}
Diabetes mellitus leads to high blood glucose due to disturbance in insulin secretion or sensitivity. Diabetes mellitus type 1 results from insufficient insulin release. Diabetes mellitus type 2 and gestational diabetes occur mainly due to insulin resistance which is associated with obesity and pregnancy. Diabetes is a chronic disease that needs three approaches for successive treatment which are food regimen (diet), exercise, and medication (either by insulin or pills). These three treatment strategies should occur together. Insulin treatment is typically used to treat type 1 diabetes, and on the occasion, that type 2 patients don't react to different drugs. Medications that don't contain insulin are given either as oral pills or by infusion. The most distinguished of these medications and the first to be utilized to treat diabetes is metformin. Doing a pancreas transplant is especially valuable for type 1 patients. There is no negative impact of diabetes on the marital relationship because high blood glucose level doesn't affect the menstrual cycle and ovulation happens normally. There is no issue with the wedding a woman with diabetes as diabetes doesn't influence the productivity of the patient, as this woman can manage her sugar level appropriately. Some diabetic women have little sexual desire but unlike diabetic men, they have no difficulties during or after sex. Controlling the blood glucose level during pregnancy is very important for the security of both the mother and the baby. Women with gestational diabetes could begin breastfeeding as non-diabetic women, however, the time of breastfeeding in women with gestational diabetes was two months not as much as that of uninfected women.
\end{abstract}

Keywords: Diabetes, Women, Health

\section{Types of diabetes mellitus}

The number of diabetics around the world reached 382 million in 2013. The number of diabetics in Egypt surpassed 11 million patients as indicated by Egyptian records, while the World Health Organization estimated the number of diabetic patients in Egypt to 7.8 million in 2015. There are three main types of diabetes which are type 1 , type 2 , and gestational diabetes. Diabetes mellitus type 1 results from insufficient insulin release. Diabetes mellitus type 2 and gestational diabetes occur mainly due to insulin resistance which is associated with obesity and pregnancy. In all types, diabetic patients suffer from high blood glucose levels, polyurea, thirst, a decline in weight, and weakness. Diabetes is accompanied by severe complications including ketonemia, coronary disease, chronic renal damage, retinal damage, peripheral neuritis, and vascular disorders, which may cause sexual shortcoming and postponed wound recuperating, particularly foot wounds that may transform into gangrene and may lead to the removal of the foot ${ }^{(1)}$. Health education about diet, exercise, and medication is the primary nursing role that is related to lower diabetes hazards.

Type I diabetes happens when insulin secretion decreased from beta cells in the islets of Langerhans in the pancreas. This mainly is a genetic disease in which an autoimmune reaction occurs. The body of type I diabetic patients forms antibodies that attack and damage the beta cells. Type 1 diabetes can affect kids "child diabetes" and adults. This type of diabetes can be treated only with insulin injection to compensate for the shortage of insulin in these patients
(2). Type 2 diabetes is the most common type of diabetes that affects many people worldwide. In this type, the insulin is secreted normally however, the cells can not sense insulin due to problems related to insulin receptors and/or intracellular insulin pathway. This type affects mainly adults from the age of 40 years and above. Obesity and metabolic syndrome are the main risk factor for this type of diabetes ${ }^{(3)}$. The blood of these patients contains a high amount of insulin and therefore the typical treatment is through pills rather than insulin injections. Most of these antidiabetic drugs (pills) act through squeezing the pancreas to secret insulin or through inhibition of glucagon (which is a hormone antagonizes the effect of insulin). Some other pills contain metformin which increases the sensitivity of insulin receptors ${ }^{(4)}$. Gestational diabetes happens in $3.5 \%$ of pregnant women. This is a temporary type of diabetes that usually disappear after labor. However, it sometimes develops to type 2 diabetes ${ }^{(5)}$.

\section{Signs and symptoms}

Manifestations of diabetes include thirst, polyurea, increased appetite (hunger), fatigue, and weight loss. In type 1 diabetes, these symptoms may grow quickly particularly in kids. However, type 2 diabetes, they develop very slowly and sometimes pass unnoticed. Increasing glucose in the blood above the renal threshold makes it hard for the kidneys to reabsorb glucose which passes out with urine causing polyurea and thirsty. High levels of glucose affect the blood supply of retina causing vision-related disorders. Other symptoms include neuritis especially for peripheral nerves and muscular fatigue ${ }^{(5)}$.

\section{Diagnosis}

With urine glucose, there is an increase in glucose in the blood, either forever or irregularly. The glucose level in fasting diabetes is 
$126 \mathrm{mg} / \mathrm{dL}$ or more and two hours after $75 \mathrm{~g}$ of oral glucose is taken (Tolerance Glucose Test) the level increases to $200 \mathrm{mg} / \mathrm{dL}$ or above. Patients who have fasting blood glucose from 100 to $125 \mathrm{mg} / \mathrm{dL}$ are called prediabetic and patients who have diabetes over $140 \mathrm{mg} / \mathrm{dL}$ and more than $200 \mathrm{mg} / \mathrm{dL}$ two hours after taking $75 \mathrm{~g}$ glucose in the mouth are called diabetic. Measurement of glycated hemoglobin (HbA1c) used to detect glucose levels in the last three months and help physicians to decide the dangers of diabetes on the kidneys and the eye ${ }^{(6)}$.

\section{Management}

Diabetes is a chronic disease that needs three approaches for successive treatment which are food regimen (diet), exercise, and medication (either by insulin or pills). These three treatment strategies should occur together. Eating healthy food of low carbohydrate and fat and high fibers contents is very important for all diabetic patients to control their higher glucose levels in the blood. Daily exercise is very crucial for diabetic patients, with at least walking for 30 minutes per day. Muscular exercise increases the sensitivity of the insulin receptor and allows muscles to uptake a large amount of glucose from the blood. Insulin treatment is typically used to treat type 1 diabetes, and on the occasion, that type 2 patients don't react to different drugs. Insulin is infused under the skin with syringes. Medications that don't contain insulin are given either as oral pills or by infusion, and they have numerous types. Some of them invigorate insulin discharge from the pancreas, including what restrains the synthesis of glucose from the liver, and some types increase the body's reaction to insulin. The most distinguished of these medications and the first to be utilized to treat diabetes is metformin. These antidiabetic drugs incorporate rosiglitazone, chlorpropamide, repaglinide, acarbose, and others. Doing a pancreas transplant is especially valuable for type 1 patients. Upon the accomplishment of this procedure, the patient doesn't have to infuse insulin once more, yet it likewise conveys a few dangers to buy the body dismissing the new organ. Accordingly, this strategy is normally performed for patients who don't react to insulin, or who will experience a kidney transplant ${ }^{(7)}$.

\section{Diabetes and marriage}

There is no negative impact of diabetes on the marital relationship because high blood glucose level doesn't affect menstrual cycle and ovulation happens normally. There is no issue with the wedding a woman with diabetes as diabetes doesn't influence the productivity of the patient, as this woman can manage her sugar level appropriately. Some diabetic women have little sexual desire but unlike diabetic men, they have no difficulties during or after sex ${ }^{(8)}$.

\section{Diabetes and pregnancy}

Controlling the blood glucose level during pregnancy is very important for the security of both the mother and the baby. Pregnancy could induce diabetes in individuals who don't have diabetes, and also could increase diabetes complications in diabetic individuals. However, diabetes doesn't lessen the chance of pregnancy, yet it contrarily influences the pregnancy and the baby, and on the mother, if the woman who has diabetes and her blood glucose gets uncontrolled. To decrease the danger of embryo premature birth, it is important to keep blood sugar at the most ideal levels during early pregnancy ${ }^{(9)}$.

Since insulin retention is common during pregnancy, a diabetic pregnant woman may need to take a larger dose of insulin. Keeping up blood glucose at suitable levels during the last trimester of pregnancy supports the development of fetal organs, bring forth an ordinary weight, and lessen the danger of hypoglycemia after birth ${ }^{(9)}$.

High levels of glucose in pregnant decrease the immunity and increase the susceptibility to preeclampsia, infections. However, if this level is controlled, pregnancy can continue as naturally as any other natural pregnant woman. The high blood glucose level in the initial three months of pregnancy induces the occurrence of fetal anomalies, and in the second three months, it expands the rate of preeclampsia and increases amniotic fluid. Diabetes also prompts hypertension and placental perfusion, which prompts the underdevelopment of the embryo. The effect of pregnancy on diabetes includes the reduction of insulin sensitivity and elevation of possibility for vascular damage of kidney and retinal capillaries (10).

\section{Breastfeeding and diabetes}

Breastfeeding can decrease a kid's danger of numerous medical issues. Women with gestational diabetes could begin breastfeeding as non-diabetic women, however, the time of breastfeeding in women with gestational diabetes was two months not as much as that of uninfected women ${ }^{(11)}$. A previous report exhibited that insulin significantly affects the regular milk development procedure of breastfeeding mother insulin expands the adequacy of the organs liable for making milk ${ }^{(12,13)}$.

\section{References}

1. Wexler DJ, Grant RW, Meigs JB, Nathan DM, Cagliero E. Sex disparities in the treatment of cardiac risk factors in patients with type 2 diabetes. Diabetes Care. 2005;28:514-20.

2. Bellamy L, Casas JP, Hingorani AD, Williams D. Type 2 diabetes mellitus after gestational diabetes: A systematic review and meta.analysis. Lancet. 2009;373:1773-9.

3. Metzger BE, Lowe LP, Dyer AR, Trimble ER, Chaovarindr U, Coustan DR, et al. for HAPO Study Cooperative Research Group. Hyperglycemia and adverse pregnancy outcomes. N Engl J Med. 2008;358:1991-2002.

4. Abdellatif KRA, Fadaly WAA, Kamel GM, Elshaier YAMM, ElMagd MA. Design, synthesis, modeling studies and biological evaluation of thiazolidine derivatives containing pyrazole core as potential anti-diabetic ppar- $\gamma$ agonists and anti-inflammatory cox-2 selective inhibitors. Bioorganic Chemistry 2019;82:86-99.

5. Billionnet C, Mitanchez D, Weill A, Nizard J, Alla F, Hartemann A, et al. Gestational diabetes and adverse perinatal outcomes from 716,152 births in France in 2012. Diabetologia. 2017;60:636-44.

6. Retnakaran R, Shah BR. Mild glucose intolerance in pregnancy and risk of cardiovascular disease: A population.based cohort study. CMAJ. 2009;181:371-6.

7. Kessous R, Shoham.Vardi I, Pariente G, Sherf M, Sheiner E. An association between gestational diabetes mellitus and long.term maternal cardiovascular morbidity. Heart. 2013;99:1118-21.

8. Ratner RE, Christophi CA, Metzger BE, Dabelea D, Bennett PH, Pi.Sunyer X, et al. Prevention of diabetes in women with a history of gestational diabetes: Effects of metformin and lifestyle interventions. J Clin Endocrinol Metab. 2008;93:4774-9.

9. Aroda VR, Christophi CA, Edelstein SL, Zhang P, Herman WH, Barrett.Connor E, et al. The effect of lifestyle intervention and metformin on preventing or delaying diabetes among women with and without gestational diabetes: The diabetes prevention program outcomes study 10.year follow.up. J Clin Endocrinol Metab. 2015;100:1646-53.

10. Bao W, Tobias DK, Bowers K, Chavarro J, Vaag A, Grunnet LG, et al. Physical activity and sedentary behaviors associated with 
risk of progression from gestational diabetes mellitus to type 2 diabetes mellitus: A prospective cohort study. JAMA Intern Med. 2014;174:1047-55.

11. Nicolaisen I. Cultural perceptions, gestational diabetes, and development. Int J Gynaecol Obstet. 2009;104(Suppl 1): S8-10.

12. Mahtab H, Habib SH. Social and economic consequences of diabetes in women from low.income countries: A case study from Bangladesh. Int J Gynaecol Obstet. 2009;104(Suppl 1): S14-6.

13. Ramachandran A, Snehalatha C, Baskar AD, Mary S, Kumar CK, Selvam S, et al. Temporal changes in the prevalence of diabetes and impaired glucose tolerance associated with lifestyle transition occurring in the rural population in India. Diabetologia. 2004;47:860-5. 